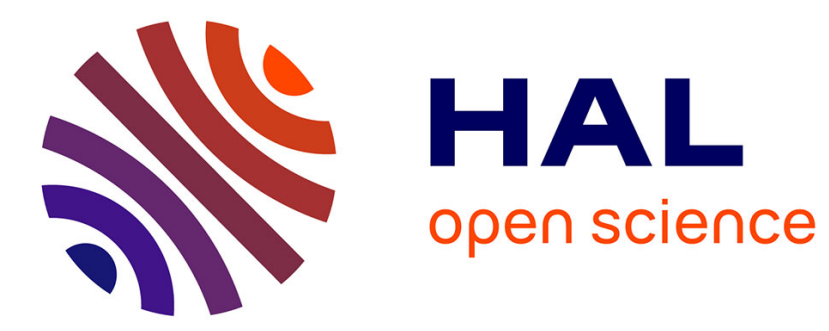

\title{
Coalescence of armored interface under impact
}

\author{
C. Planchette, A.-L. Biance, Olivier Pitois, Elise Lorenceau
}

\section{To cite this version:}

C. Planchette, A.-L. Biance, Olivier Pitois, Elise Lorenceau. Coalescence of armored interface under impact. Physics of Fluids, 2013, 25 (4), pp.042104. 10.1063/1.4801320 . hal-00946076

\section{HAL Id: hal-00946076 \\ https://hal-enpc.archives-ouvertes.fr/hal-00946076}

Submitted on 3 Sep 2015

HAL is a multi-disciplinary open access archive for the deposit and dissemination of scientific research documents, whether they are published or not. The documents may come from teaching and research institutions in France or abroad, or from public or private research centers.
L'archive ouverte pluridisciplinaire HAL, est destinée au dépôt et à la diffusion de documents scientifiques de niveau recherche, publiés ou non, émanant des établissements d'enseignement et de recherche français ou étrangers, des laboratoires publics ou privés. 


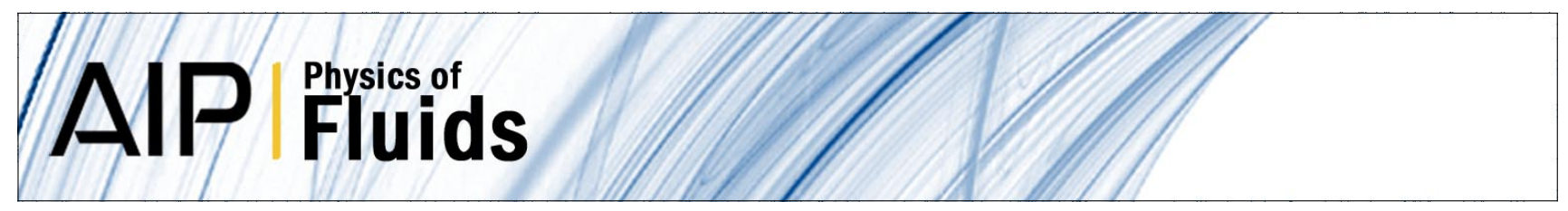

\section{Coalescence of armored interface under impact}

C. Planchette, A.-L. Biance, O. Pitois, and E. Lorenceau

Citation: Phys. Fluids 25, 042104 (2013); doi: 10.1063/1.4801320

View online: http://dx.doi.org/10.1063/1.4801320

View Table of Contents: http://pof.aip.org/resource/1/PHFLE6/v25/i4

Published by the American Institute of Physics.

\section{Additional information on Phys. Fluids}

Journal Homepage: http://pof.aip.org/

Journal Information: http://pof.aip.org/about/about_the_journal

Top downloads: http://pof.aip.org/features/most_downloaded

Information for Authors: http://pof.aip.org/authors

\section{ADVERTISEMENT}

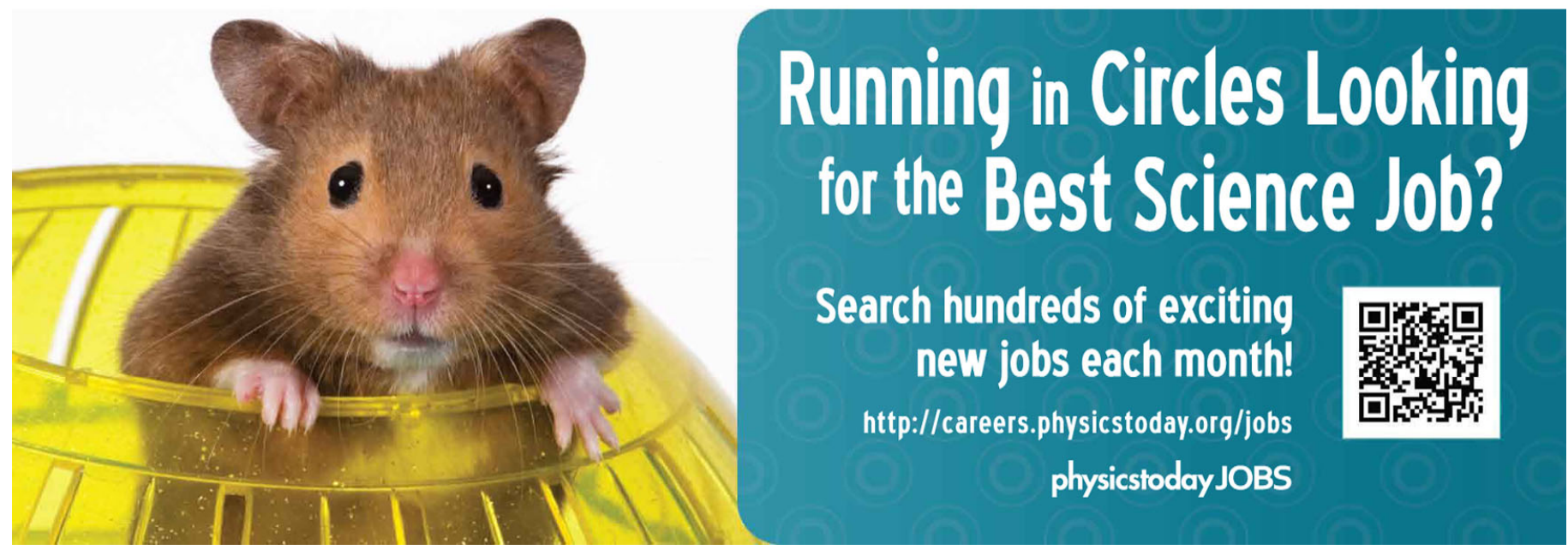




\title{
Coalescence of armored interface under impact
}

\author{
C. Planchette, ${ }^{1,2}$ A.-L. Biance, ${ }^{3}$ O. Pitois, ${ }^{4}$ and E. Lorenceau ${ }^{4}$ \\ ${ }^{1}$ LPMDI, Université Paris-Est Marne-La-Vallée, FRE3300 CNRS, 5 Bd Descartes, \\ 77454 Marne-la-Vallée, France \\ ${ }^{2}$ Research Center Pharmaceutical Engineering, Inffeldgasse 13, A-8010 Graz, Austria \\ ${ }^{3}$ Institut Lumière Matière, Université de Lyon, UMR5306 Université Lyon 1-CNRS, \\ 69622 Villeurbanne, France \\ ${ }^{4}$ Laboratoire Navier, Université Paris-Est, UMR8205 CNRS-ENPC-IFSTTAR, 2 allée Kepler, \\ 77420 Champs-sur-Marne, France
}

(Received 15 May 2012; accepted 19 March 2013; published online 23 April 2013)

\begin{abstract}
Armored interfaces refer to fluid interfaces on which a compact monolayer of particles is adsorbed. In this paper, we probe their robustness under impact. For such an investigation, the impact of a drop (covered or not by particles) on a flat armored interface is considered. Two regimes are observed: small drops impacting at low velocities do not coalesce, while bigger drops falling at higher velocities lead to coalescence. The coalescence which occurs when the impacting drop has just reached its maximum extension directly results from the formation of bare regions within the armor. We therefore propose a geometric criterion to describe this transition. This simple modeling is able to capture the dependence of the measured velocity threshold with particle size and drop diameter. The additional robustness experienced by double armors (both drop and puddle covered) results in an increase of the measured velocity threshold, which is quantitatively predicted. (c)2013 AIP Publishing LLC. [http://dx.doi.org/10.1063/1.4801320]
\end{abstract}

\section{INTRODUCTION}

The study of armored interfaces, which refer to all kinds of interfaces on which a compact monolayer of particles is adsorbed, dates back to the early 1900s. ${ }^{1,2}$ Such armors can be achieved at a liquid/liquid interface or an air/liquid interface, where the particles provide a coating for an individual bubble ${ }^{3-8}$ or a water drop. ${ }^{9-11}$ They are also used to stabilize large collections of entities, such as particle stabilized foams ${ }^{12-17}$ or dry water. ${ }^{13,18-20}$ Most of the potential applications involving these objects stem from the mechanical isolation properties of such layers: ${ }^{11,21-24}$ small liquid quantities can be moved on any substrate without any pollution or loss. In these examples, which often involve a large number of entities, the resistance to coalescence between two adjacent liquid marbles or coated bubbles is crucial. Coalescence can occur when the two interfacesinitially separated by a thin film of air or liquid decorated with the solid particles-connect. This suggests that the liquid menisci between the particles of the layer are not stable and slowly move along the particles. The maximum pressure of stability of a meniscus is proportional to the local mean curvature of the liquid/air interface inside the pores. It can be evaluated as with porosimetry techniques, considering that the particles are irreversibly bound to the interfaces and remain immobile during the whole coalescence process. ${ }^{17,25-28}$ Yet, coalescence between bubbles or drops is generally mediated by significant deformation of the interface ${ }^{29}$ allowing the particles to rearrange along the surface during the contact. This suggests a strong coupling between the interface deformation and the particle motion as already highlighted in quasistatic situations where the two interfaces are gently brought into contact. ${ }^{30-32}$

Here, we investigate the coalescence mechanism of armored interfaces focusing on the local interface deformation and the subsequent microscopic movement of the particles. We first consider the impact of a bare drop on a puddle armored with a single layer of particles (single armor case), then the impact of an armored drop on an armored puddle (double armor case). In both cases, two 
regimes are observed: at high velocity, coalescence occurs while at low velocity the coalescence is inhibited by the presence of the particles. We measure the coalescence threshold velocity and propose a quantitative understanding of our results.

\section{EXPERIMENTS}

\section{A. Particles}

The particles are spherical glass beads of density $\rho_{p}=2200 \mathrm{~kg} / \mathrm{m}^{3}$. To obtain several sets of monodisperse particles with the same contact angle, we proceed as follows. First, a large amount of particles with a broad size distribution are chemically modified by silanization to make them hydrophobic. ${ }^{33}$ The silanization is performed on dry particles that have been cleaned with a piranha mixture (70\% of concentrated sulfuric acid to $30 \%$ hydrogen peroxide solution). For $100 \mathrm{~g}$ of particles, we use $100 \mathrm{ml}$ of a solution of FDTCS (Perfluorodecyltrichlorosilane ABCR GmbH \& Co. KG) in toluene at $0.05 \%$. After $20 \mathrm{~min}$, the reaction is stopped with ethanol, the particles are rinsed and dried. Then, we sieve all the particles that have received the same chemical treatment in order to approach monodisperse distributions. After sieving, we obtain nine sets of particles, each of them having a sharp gaussian distribution. The mean diameter $d$ and the standard deviation $\sigma$ of the different sets are reported in Table I. These distributions are obtained by image processing of particle pictures at an appropriate magnification. More precisely, particles are spread far below the monolayer density onto glass plates with back lighting. The shadow area of each particle is measured using the threshold function of the ImageJ software and is then converted into the corresponding particle diameter. For each set, 1000 to 2000 particles are analyzed. The last step consists in measuring the contact angle of the particles from side view microscope pictures by placing a single particle at the air/water interface of a pendant drop. The contact angle $\theta$ is found to be equal to $112^{\circ}$ for every monodisperse set. Systematically, 20 contact angle measurements are performed resulting in a typical standard deviation of $5^{\circ}$.

\section{B. Preparation of the armored interfaces and experimental set-up}

To obtain a flat armored liquid interface, we force several liquid marbles to coalesce by compressing them together. Each liquid marble is obtained by rolling a water drop onto a bed of dry particles. ${ }^{9,10}$ After coalescence, the resulting object, flattened by gravity, is an armored puddle that does not wet the surface thanks to the particle layer as sketched in Fig. 1. Its diameter varies between 2 and $3 \mathrm{~cm}$, while its height is $h=4.3 \mathrm{~mm}$. The thickness of a gravitary puddle in a non-wetting situation is $2 \kappa^{-1}$, where the capillary length $\kappa^{-1}=\sqrt{\gamma_{e} / \rho g}$ depends on the effective surface tension $\gamma_{e}$ of the liquid/air interface and the liquid density $\rho$. This typically results in $\gamma_{e}=52 \mathrm{mN} / \mathrm{m}$ when taking $\rho=1000 \mathrm{~kg} / \mathrm{m}^{-3}$. This is in quantitative agreement with observations in the literature as the dense particle monolayer at the interface is known to lower the surface tension. ${ }^{10,23,34,35}$ We control

TABLE I. Size distributions of the particle lots used for our experiments.

\begin{tabular}{cc}
\hline \hline $\begin{array}{c}\text { Particle diameter } \\
d(\mu \mathrm{m})\end{array}$ & $\begin{array}{c}\text { Standard deviation } \\
\sigma(\mu \mathrm{m})\end{array}$ \\
\hline 32 & 4 \\
40 & 7 \\
43 & 5.5 \\
80 & 3 \\
86 & 4.5 \\
95 & 7 \\
134 & 4.5 \\
146 & 4.5 \\
159 & 11 \\
\hline \hline
\end{tabular}



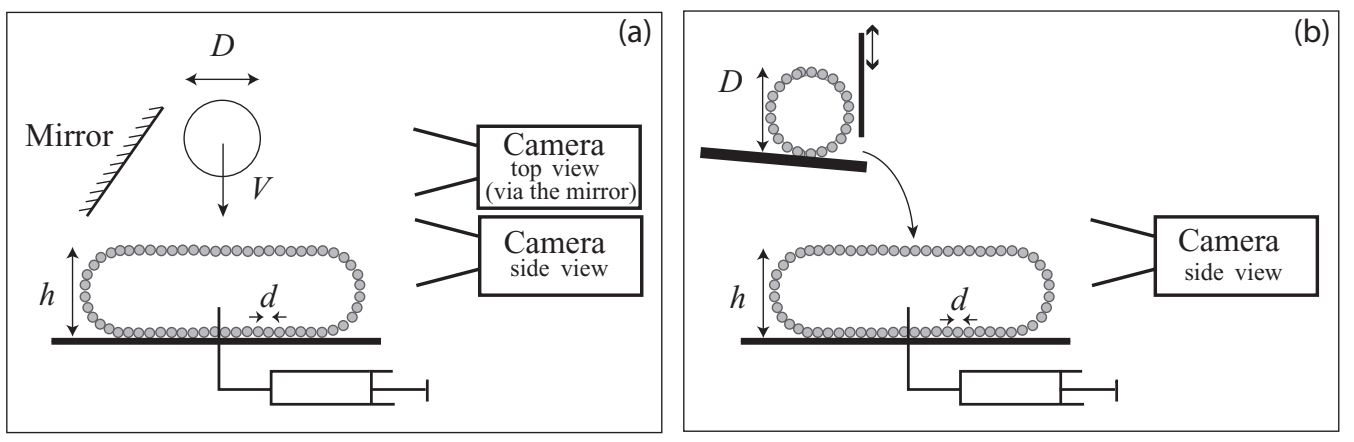

FIG. 1. (a) Single armor experimental set-up: a bare water drop impacts on a water puddle armored with particles of diameter $d$. Two synchronized high speed cameras record side and top views of the impacts. (b) Double armor experimental set-up: an armored water drop impacts on armored interfaces. A high speed camera records a side view of the impacts.

$\phi_{s}$, the surface density of the particle monolayer defined as the surface occupied by the particles per unit of area, by pumping and injecting water in the puddle via a syringe placed below it, as sketched in Fig. 1. With this set-up, we can adjust the puddle surface while keeping constant the number of adsorbed particles. This results in a good control of the surface density which is measured by image analysis of the surface. ${ }^{35}$ Knowing the particles' diameter, we find that the surface density $\phi$ ranges between 0.95 and 0.98 . This value is larger than the jamming density of a compact monolayer $\phi_{j}=\frac{\pi}{2 \sqrt{3}} \approx 0.91$ and suggests that the particles locally overlap as no macroscopic wrinkle can be seen on the puddle surface.

We first consider a single armor situation that is the impact of a bare water drop on an armored liquid puddle prepared as described above. $D$, the drop diameter, typically ranges from 2 to $4 \mathrm{~mm}$, while $V$, the impacting velocity, is adjusted between $0.3 \mathrm{~m} / \mathrm{s}$ and $2 \mathrm{~m} / \mathrm{s}$ by varying the drop dispenser height. Consequently, the Weber number $\left(W e=\rho V^{2} D / \gamma\right)$ associated to the bare drop and evaluated taking $\gamma=72 \mathrm{mN} / \mathrm{m}$ and $\rho=1000 \mathrm{~kg} / \mathrm{m}^{3}$, varies between 1 and 250 . The impact is recorded using two high-speed cameras as shown in Fig. 1(a). The two cameras are triggered simultaneously and record, respectively, at $923 \mathrm{fps}$ for the side view and $3500 \mathrm{fps}$ for the top view. We deduce the drop size and velocity from the side view and collect information about the coalescence mechanism from the top view.

In the double armor experiments, an armored liquid marble impacts on an armored liquid puddle. There, the liquid marble is prepared by rolling a water drop onto a bed of dry particles, then it is placed on a launching platform of variable height as depicted in Fig. 1(b). The impact is recorded with a single high speed camera as the drop is no longer transparent due to its particle armor.

\section{RESULTS AND DISCUSSION}

\section{A. Experimental results}

We observe two different regimes as reported in Figs. 2 and 3 where side and top views of bare drops impacting an armored puddle at different velocities are exhibited. Figure 2(a) illustrates the impact of a relatively slow drop on the armored interface. The drop first flattens at its bottom, then exhibits a classical staircase pyramidal shape ${ }^{36}$ and is transiently engulfed below the puddle surface before reappearing to eventually sit on the interface.

Several small holes, with a typical size of hundreds of microns, appear in the armor at the edge of the drop as emphasized by the arrows in Fig. 2(a). These holes do not lead to coalescence. Figure 3(b) (side view) shows the deformation of the impacting drop prior to the coalescence with the armored puddle. Here again, the drop first flattens at its bottom, then also exhibits a staircase pyramidal shape, but is irremediably swallowed by the large puddle. Figure 3(a) (top view) illustrates the modification of the particle layer density induced by the same impact. The shock breaks up the armored interface which disrupts in several points at the edge of the drop as highlighted by the arrows. Then, a large 

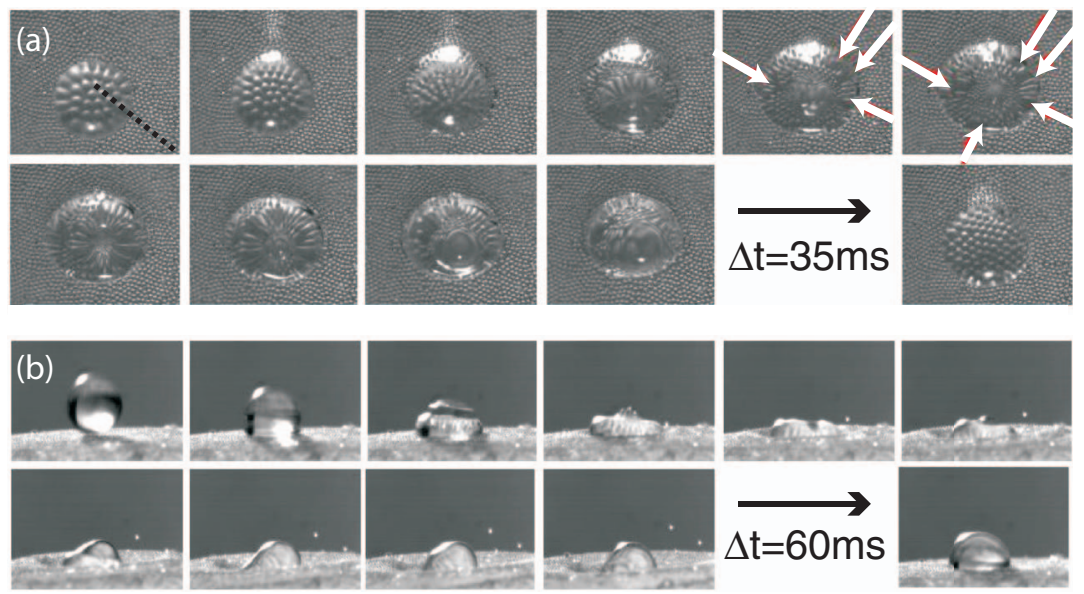

FIG. 2. Top and side views of a drop $(D=2.55 \mathrm{~mm})$ impacting on an armored interface $(d=146 \mu \mathrm{m})$ with a velocity $V=0.44 \mathrm{~m} / \mathrm{s}$. This does not lead to the coalescence of the drop with the bath. The time interval between two consecutive pictures is $667 \mu \mathrm{s}$ for the top view (a) and $1.1 \mathrm{~ms}$ for the side view (b) except for the last images for which the time interval is $35 \mathrm{~ms}$ for the top view (a) and $60 \mathrm{~ms}$ for the side view (b). In (a) holes between the particles can be observed in the periphery of the drop close to its maximal extension as highlighted by the arrows. The dotted line in (a) is the reference line used to perform the spatio-temporal analysis displayed in Fig. 4.

hole opens through which the drop sinks into the puddle. After the coalescence, the interface heals and no scarring is observed in the armor (see last image in Fig. 3(a)).

To obtain a quantitative description of the relative motion of the particles during the impact, we perform analysis of images such as those of Figs. 2(a) and 3(a). The spatio-temporal diagrams of Fig. 4 showing the time evolution of the pixel values along the dotted lines of Figs. 2(a) and 3(a) highlight the motion of the individual particles during the impact. Before the impact, the particles do not move as shown by the bright straight vertical lines. Then, impact occurs as illustrated by the points A in Figs. 4(a) and 4(b). Consecutively, a wave, which propagates along
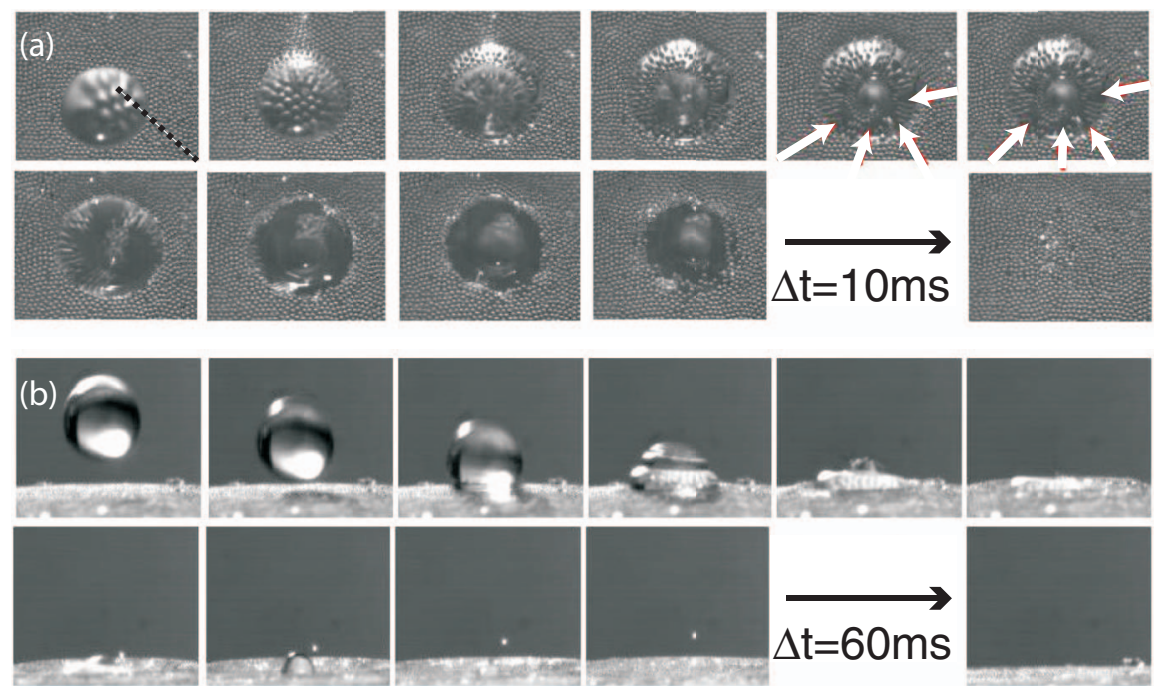

FIG. 3. Top and side views of a drop $(D=2.55 \mathrm{~mm})$ impacting on an armored interface $(d=146 \mu \mathrm{m})$ with a velocity $V=0.60 \mathrm{~m} / \mathrm{s}$. The time interval between two consecutive pictures is $667 \mu$ s for the top view (a) and $1.1 \mathrm{~ms}$ for the side view (b) except for the last images for which the time interval is $10 \mathrm{~ms}$ for the top view (a) and $60 \mathrm{~ms}$ for the side view (b). This impact leads to the coalescence of the drop with the bath. In (a) holes between the particles can be observed in the periphery of the drop close to its maximal extension as highlighted by the arrows. The dotted line in (a) is the reference line used to perform the spatio-temporal analysis displayed in Fig. 4. 


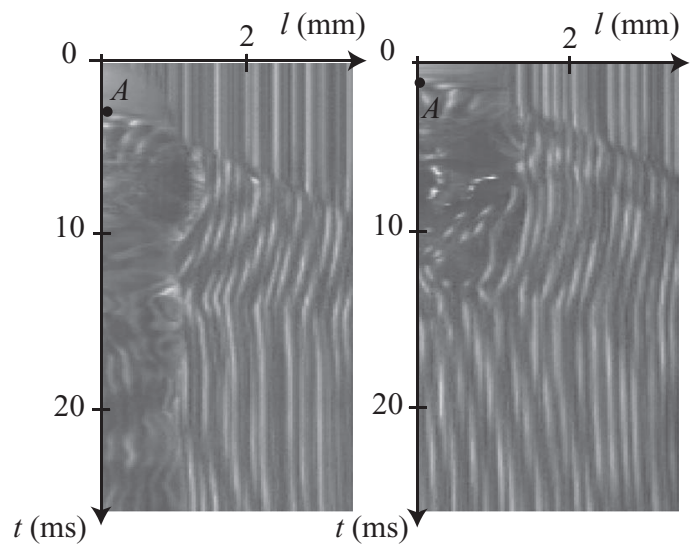

FIG. 4. Spatio-temporal diagram, which illustrates the motion of the particles along the lines drawn in Figs. 2(a) and 3(a).

the surface at a constant velocity equal to $0.5 \mathrm{~ms}^{-1}$ displaces the particles of $146 \mu \mathrm{m}$. Then, all the particles oscillate in phase with the oscillating drop. This is not true for the particles in the vicinity of the coalescence zone as observed in Fig. 3(b). In this region, the particles rapidly move inward to close the hole induced by the coalescence: the bare surface with a diameter of $2.5 \mathrm{~mm}$ is healed in $8 \mathrm{~ms}$. This suggests a velocity of $0.15 \mathrm{~m} \cdot \mathrm{s}^{-1}$. A quantitative understanding of what sets this value would provide interesting features concerning the particles surface pressure and the surface flow due to the coalescence process. However, light refraction in this highly curved region generates image distortion. A correct measurement of the healing velocity would therefore require one to know precisely the shape of the drop during the whole impact process, which is far beyond the scope of this paper.

Now that we have observed that particles directly follow droplet distortion, we investigate when coalescence occurs. Indeed, for drops of a given size, the transition between these two regimes corresponds to a threshold velocity $V_{1}$. Drops impacting slower than $V_{1}$ do not coalesce with the armored puddle while faster drops do. We define the threshold velocity as the average between the highest observed non-coalescence velocity and the lowest velocity where coalescence occurs. The uncertainty of the measurement is $\pm 0.02 \mathrm{~m} / \mathrm{s}$. $V_{1}$ is reported as a function of the drop diameter $D$ for two particle diameters $(d=134 \mu \mathrm{m}$ and $d=159 \mu \mathrm{m})$ on Fig. 5(a). In both cases, the variations of $V_{1}$ with $D$ are similar: $V_{1}$ decreases when $D$ increases following a power law close to $V_{1} \sim D^{-0.8}$. This holds as long as the drop diameter is smaller than $3 \mathrm{~mm}$. For larger drops, $V_{1}$ does not depend on the droplet diameter which may be caused by the limited puddle thickness $h=4.3 \mathrm{~mm}$ as discussed hereafter. The influence of the particle diameter $d$ on $V_{1}$ is presented in Fig. 5(b). The
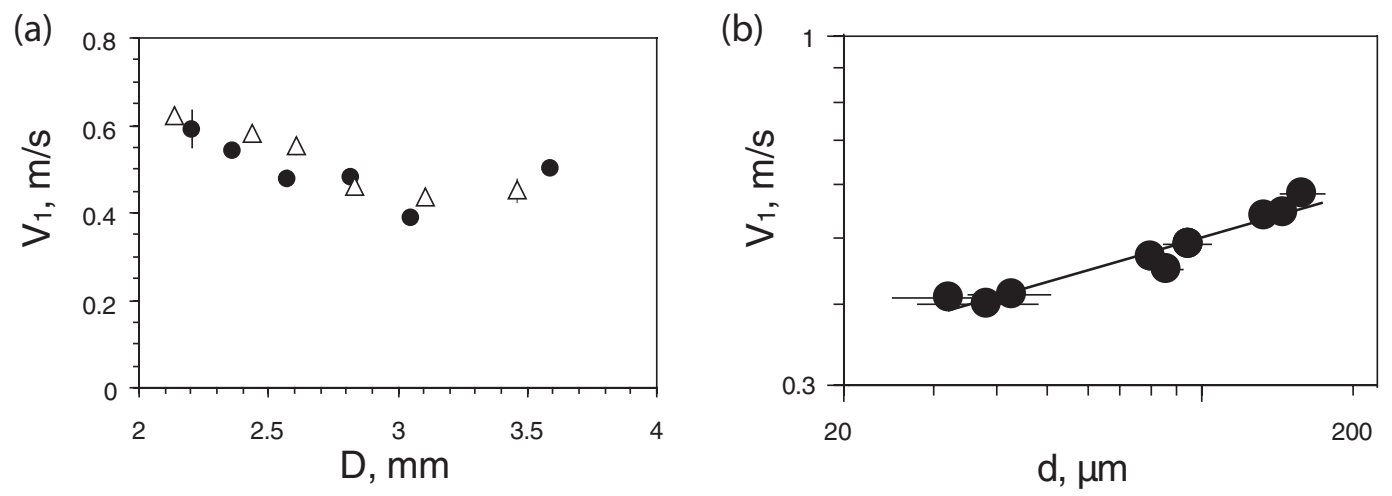

FIG. 5. (a) Evolution of $V_{1}$ with $D$ for two armored puddles with $d=134$ and $d=159 \mu \mathrm{m}$. For $D<3 \mathrm{~mm}, V_{1}$ decreases with $D$. (b) Evolution of $V_{1}$ with $d$ for a given drop diameter $(2.5 \mathrm{~mm}), V_{1}$ increases with $d$. The solid line indicates $V_{1}=0.2 d^{0.22}$. 
trend previously observed for $d=134 \mu \mathrm{m}$ and $d=159 \mu \mathrm{m}$ is confirmed: $V_{1}$ increases with $d$ as $V_{1} \simeq 0.2 d^{0.22}$. Similar results are observed for the double armor case.

\section{B. Single armor coalescence}

The rupture of interfaces armored with hydrophobic particles has already been investigated by considering the impact of a liquid marble on a solid motionless surface. ${ }^{10,37}$ During an impact, the liquid marble flattens and spreads on the solid media. The surface area of the impacting dropinitially spherical - is suddenly increased. Consequently, the dense coating layer is disrupted and contact between the liquid and the solid surfaces can establish if the damage in the protective layer is severe enough. The wetting liquid thus adheres to the surface rather than recoiling into a single armored drop. A threshold velocity above which the encapsulated liquid wets the solid surface has been measured for different types of particle of various sizes. A quantitative interpretation of this threshold based on (i) the conversion of the drop kinetic energy into surface energy and (ii) the opening of a hole of a critical size in the armor has been proposed and successfully tested. ${ }^{10,37}$ In this paper, we use a similar framework and propose a quantitative criterion for the threshold velocity of coalescence of a deformable drop on a deformable armored puddle.

In the situation considered here, the topologies of both the impacting drop and the armored puddle change as the drop's kinetic energy is distributed between the surface energies of the drop and the puddle. We first consider the deformation of the liquid drop under impact on the armored liquid puddle. We note $D_{\max }$ the diameter of the bare drop when reaching its maximal extension. $D_{\max }$ can be estimated from the top view of the impact (Figs. 2(a) and 3(a)). $D_{\max } / D$ is measured for different drop and particle diameters and is reported in Fig. 6 as a function of the Weber number $\left(W e=\rho V^{2} D / \gamma\right)$. It is almost constant and lies between 1.1 and 1.3, which corresponds to a deformation smaller than $30 \%$. Results also show an effect of particle diameter: the smaller the $d$, the smaller the $D_{\max }$.

To understand these observations, we consider the shape of a static bubble (or liquid drop) at a liquid/air (or liquid/liquid) interface with no contact angle. The shape of such a bubble deviates from a perfect sphere and can be computed as a function of the Bond number ${ }^{38,39}\left(B o=\left(\rho-\rho_{\text {air }}\right) g D^{2} / \gamma\right)$. At small $B o$, the bubbles are quasi-spheres while at large $B o$, the bubbles take a hemispherical shape. In this limit, volume conservation ensures $D_{\max } / D=2^{1 / 3} \approx 1.26$. Our dynamical data can be compared to this static analysis, introducing an effective gravity $a \sim V^{2} / D$ which is the acceleration experienced by the drop during impact. ${ }^{40}$ Replacing straightforwardly $g$ by $a$ in $B o$ yields an effective Bond number $B o^{*}=\left(\rho-\rho_{\text {air }}\right) V^{2} D / \gamma$ which is equivalent to $W e$ since $\rho>>\rho_{\text {air }}$. Note that the comparison is only qualitative as the range of velocities investigated here is limited to $V<V_{1}$. The expression of $D_{\max } / D$ computed in Ref. 39 is plotted with a thin plain line in Fig. 6 for comparison.

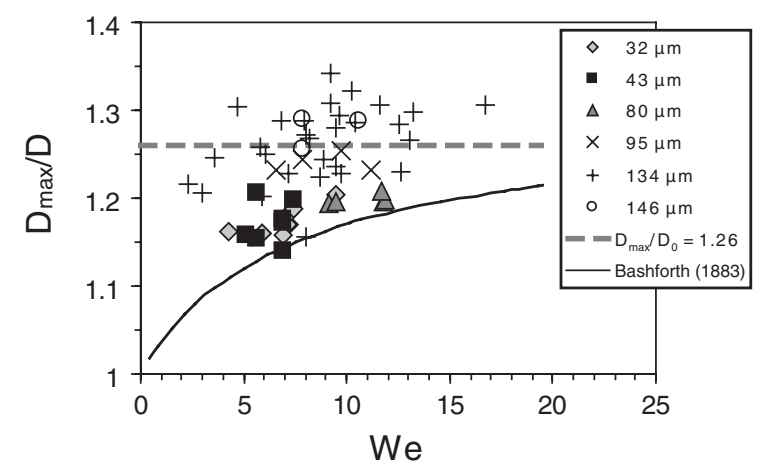

FIG. 6. Evolution of $D_{\max } / D$ with $W e=\rho V^{2} D / \gamma$ for drop diameters ranging between 2 and $3 \mathrm{~mm}$ and particle diameters between 32 and $146 \mu \mathrm{m}$. The thick black line corresponds to the maximal diameter of a quiescent bubble at a liquid air-interface as given in Ref. 39. The dashed grey line corresponds to the limit where the initial spherical drop takes a hemispherical shape. 

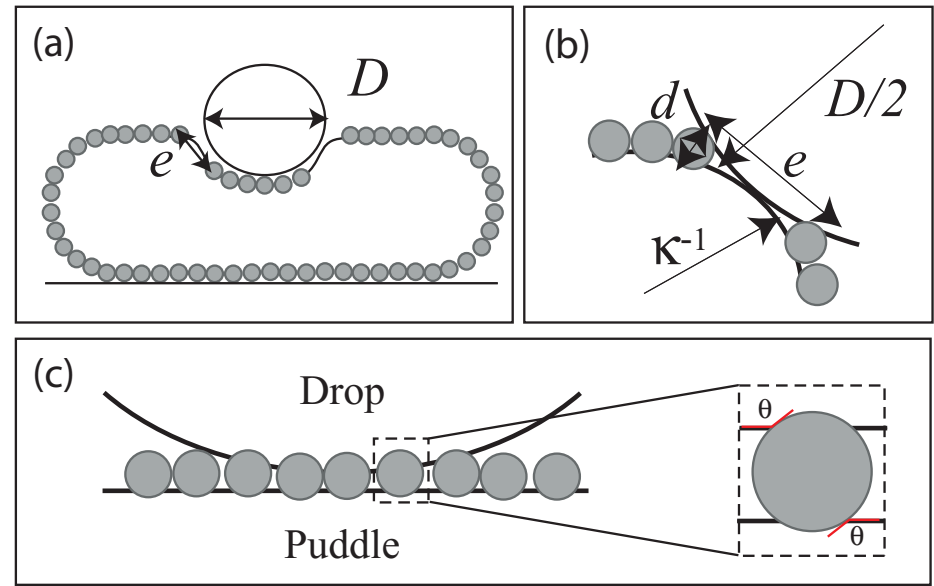

FIG. 7. (a) and (b) Typical dimensions and shape of the crater formed in the puddle by the impacting drop. (c) Schematic representation of the hydrophobic particles located between the impacting drop and the armored puddle. Satisfying the wetting conditions on both poles, the particles insure a bridge between the drop and the puddle.

Our data are in reasonable agreement with those corresponding to static lenses floating on liquid surfaces, yet, they systematically are above this line. This may be due to the limited depth of the tank, which yields a larger value of $D_{\max } / D$. The systematic variation of $D_{\max } / D$ with $d$ may be explained considering the additional energetic cost associated to the deformation of the armored layer. Such a layer experiences a bending modulus $B$ scaling with the square of the particle diameter. ${ }^{35,41}$ The energetic cost associated with interface deformation thus increases with the particle diameter. Consequently, the effective stiffness of the puddle interface increases with particle diameters, while the bare droplet stiffness is constant. ${ }^{42}$

Therefore, the smaller the particles, the more the puddle is prone to deformation, which yields a smaller value of $D_{\max } / D$. Note however that this effect is of second order since the difference in $D_{\max } / D$ between the smallest and largest particles is at most $15 \%$.

We eventually conclude that the impacting drop experiences a small deformation $\left(D_{\max }-D\right.$ always smaller than 30\%). Much of the initial kinetic energy is thus converted into surface energy via the armored puddle deformation, resulting at first order in $D_{\max } \sim D$.

We now focus on the deformation experienced by the liquid puddle. Figures 2 and 3 show that a hole, located at the edge of the impacting drop, opens in the interface. This suggests that the cavity created in the coated puddle by the impacting drop is shaped as sketched in Figs. 7(a) and 7(b) with a diameter $D_{\max }$ and a width $e$. Thus, the energy balance of the impact writes at first order

$$
\rho D^{3} V^{2} \sim \gamma D e .
$$

The next step consists in elaborating a criterion for the coalescence. To our understanding, the coalescence occurs as soon as the surface of the bare drop enters into contact with the bare air/water interface of the puddle. Hence, one may wonder what is the critical width of the hole leading to the coalescence. We propose a simple criterion based on the steric repulsion ensured by the solid particles to prevent the two curved air/water interfaces from touching each other. This argument can be understood considering Fig. 7(b): the particles prevent contact between the two interfaces only if they are large and close enough and provided that the curvatures of the two interfaces in the radial direction are not too large. In the limit of small deformation, the curvature of the impacting drop is $2 / D$. To determine the puddle interface curvature, we emphasize that the coalescence occurs when the drop reaches its maximal extension, thus when the velocity of the impacting drop reaches zero. At this particular instant, the interface is mainly shaped by capillarity and gravity, suggesting that 
the radial curvature of the interface in the vicinity of the drop is of the order of one over the capillary length $\left(\kappa^{-1}=\sqrt{\frac{\gamma}{\rho g}}\right)$.

The particles provide an efficient protection toward coalescence if $d>(\kappa+2 / D) e^{2} / 2$ (Fig. 7(b)), leading the critical width $e^{*}$ to

$$
e^{*} \sim 2\left(\frac{d D}{2+\kappa D}\right)^{1 / 2} .
$$

With $d=90 \mu \mathrm{m}, D=2 \mathrm{~mm}$, and $\kappa^{-1}=2.3 \mathrm{~mm} e^{*}$ is equal to $500 \mu \mathrm{m}$, a value in agreement with the observations of Fig. 2.

Combining Eqs. (1) and (2) yields to the following expression for the threshold velocity:

$$
V_{1} \sim\left(\frac{\gamma}{\rho D}\right)^{1 / 2}\left(\frac{d / D}{2+\kappa D}\right)^{1 / 4}
$$

The scaling provided by Eq. (3) is in reasonable agreement with our experimental observations as the dependency with $d$ is $\frac{1}{4}$ close to the experimental value 0.22 (Fig. 5(b)). It also exhibits a reasonable dependency with the drop diameter: Eq. (3) is a decreasing function of $D$ with an exponent varying from -0.75 when $D \ll \kappa^{-1}$ to -1 when $D \gg \kappa^{-1}$. In between, the exponent takes intermediate values and for $\kappa^{-1}=2.35 \mathrm{~mm}, d=100 \mu \mathrm{m}$ and $1 \mathrm{~mm}<D<4 \mathrm{~mm}$, the variation with $D$ is well fitted by a power law with an exponent of -0.82 in excellent agreement with the -0.8 of the experimental data (Fig. 5(a)). Then, a good collapse of the experimental data for $32 \mu \mathrm{m}<d$ $<159 \mu \mathrm{m}$ and $1.8 \mathrm{~mm}<D<4 \mathrm{~mm}$ is observed when plotting the experimental results as a function of $\left(\frac{\gamma}{\rho D}\right)^{1 / 2}\left(\frac{d / D}{2+\kappa D}\right)^{1 / 4}$ (see Fig. 8).

We stress that this agreement is not only qualitative but almost quantitative: when taking into account the numerical factors, we find a slope of $\sqrt{24} \sim 5$ which is close to the experimental value of 7.9. Our analysis is geometrical in nature and suggests that the whole dynamic of the system is set by the impact phenomena. In particular, it neither takes into account the specific dynamic of the armored interface nor the relative motion of the particles. The validity of this analysis is inferred by Fig. 4: in our experimental conditions, the particles move all together and instantaneously adapt to the motion of the interface as long as they are far away from the coalescence region. This is not always the case: fractures — a typical feature of a solid—can propagate on a layer of densely packed particles trapped at a liquid-gas interface under Marangoni stresses, as shown in Refs. 43 and 44. Our analysis also suggests that the incident kinetic energy liberated by the impact is mainly converted into surface energy of the raft. In particular, we do not consider the dissipation mechanisms inherent

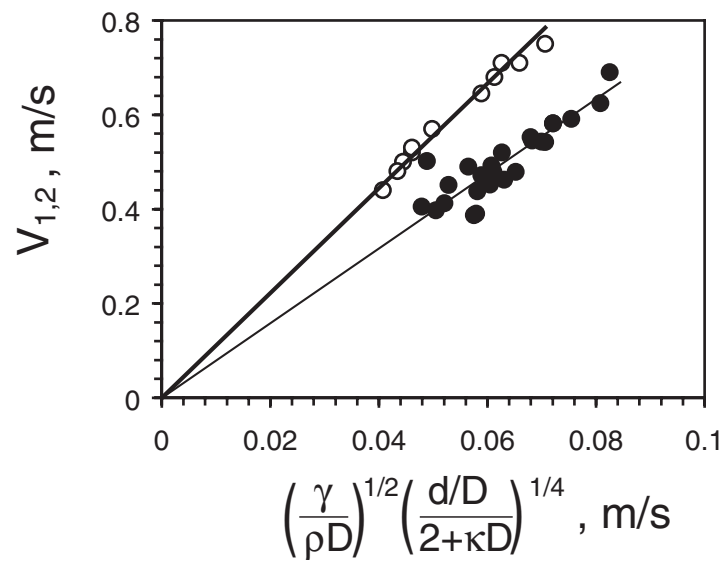

FIG. 8. Comparison of $V_{1}$ and $V_{2}$, the experimental threshold velocities for single and double armor, with the expression given by Eq. (3). The agreement is very good as the data involving all the particles listed in Table I and drop diameter ranging from $1.8 \mathrm{~mm}$ to more than $4 \mathrm{~mm}$ collapse. 
in this motion, which can either occur in a Blasius boundary layer below the particle layer or in lubrication flows in the spaces between the particles as discussed in Ref. 44. To discriminate between those two mechanisms, it would be interesting to increase the viscosity of the armored puddle. In particular, in the limit of large viscosity, we expect that the two types of dissipation do not exhibit the same dependency with the particle size.

\section{Double armor coalescence}

We now consider the situation of a double armor (depicted in Fig. 1(b)) where an armored droplet impacts an armored puddle. As for the single armor, two regimes of coalescence and noncoalescence are observed. The transition between these two regimes determines a second threshold velocity $V_{2}$, that depends a priori on $D$ and $d$. These two regimes exhibit identical features to the single armor impact. For slow impact velocity $V<V_{2}$, the droplet impacts the liquid surface, is transiently swallowed by the puddle, and eventually re-emerges out of the liquid as illustrated in Fig. 9(a). For higher impact velocity $V>V_{2}$, the two interfaces irrevocably merge as can be seen in Fig. 9(b). It seems worthwhile to quantitatively evaluate whether there is any gain in double armoring the interfaces compared to single armoring. Our measurements show that $V_{2}$ is always higher than $V_{1}$ : for $d=95 \mu \mathrm{m}$ and $D=2.5 \mathrm{~mm}$, we find $V_{1}=0.49 \mathrm{~m} / \mathrm{s}$ and $V_{2}=0.68 \mathrm{~m} / \mathrm{s}$. This difference corresponds to a significant increase in kinetic energy since $(0.68 / 0.49)^{2}=1.9$.

We use the framework developed above to understand this result. When the armored drop impacts on the armored liquid puddle, its kinetic energy is mainly converted in deformation of the armored puddle. This rapid extension of the puddle surface area generates holes in the first particle armor. However, here, the contact between the drop and the puddle is still avoided thanks to the second armor of particles around the drop. The particles of this second armor are confined between the two interfaces and tend to satisfy simultaneously the wetting conditions on both poles as sketched in Fig. 7(c). Consequently, the particles which were initially wetting the drop liquid interface with a static contact angle of $\theta$, adsorb on the puddle until the static contact angle $\theta$ between the gas, the puddle interface, and the solid is reached, thus irremediably bridging the two interfaces.

The first consequence of this "bridging" effect which was already reported in Refs. 17, 27, and 45-48 is to stick the water drop in the armored puddle where it impacted. This is demonstrated when comparing the impacts of both a drop and a glass bead on armored interfaces. After the impact, the drop neither rolls nor bounces but remains at the place where it first impacted (see Fig. 2). In this case, the drop and the puddle are stuck together via the particles confined between the two interfaces. On the contrary, a glass bead can bounce off the surface of the armored puddle as seen in Fig. 10. As the particles cannot adsorb on the solid surface of the bead, there is no adhesion.
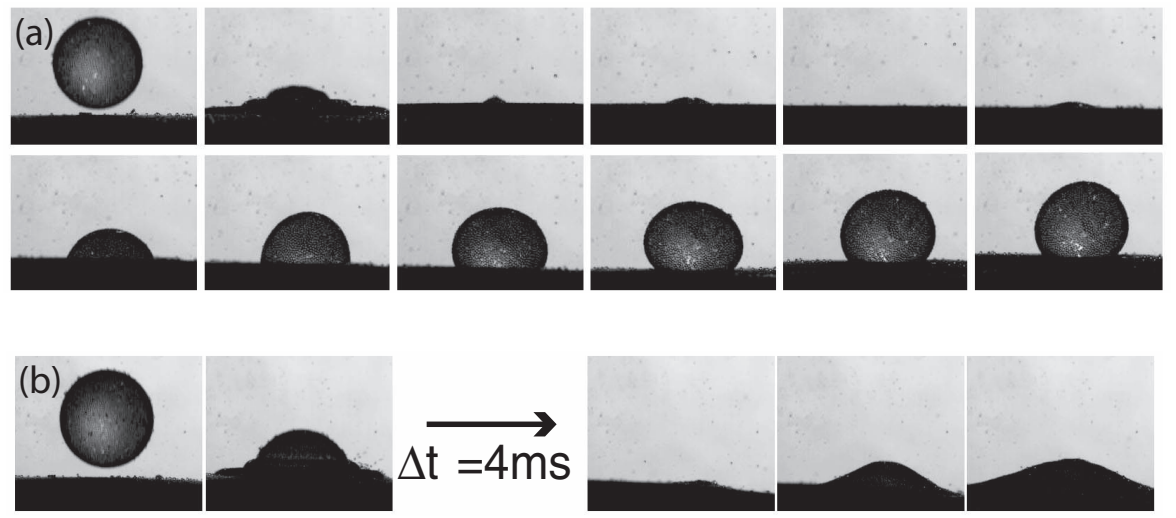

FIG. 9. (a) Side view of a liquid marble impacting on an armored interface $(\mathrm{d}=95 \mu \mathrm{m})$. The time interval between two consecutive pictures is $0.5 \mathrm{~ms}$. (a) The impact of the drop $(D=3.4 \mathrm{~mm}, V=0.49 \mathrm{~m} / \mathrm{s})$ leads to non-coalescence. (b) The impact of the drop $(D=4.1 \mathrm{~mm}, V=0.48 \mathrm{~m} / \mathrm{s})$ leads to coalescence. 


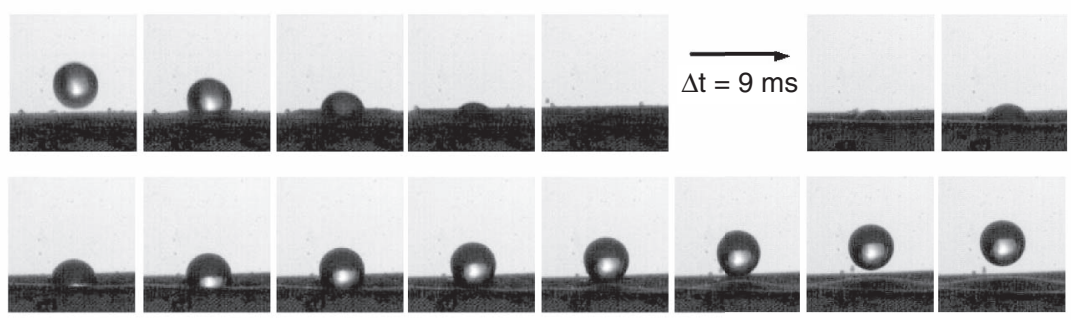

FIG. 10. Side view of a glass bead impacting on an armored puddle ( $1 \mathrm{~ms}$ elapses between two pictures). Contrary to a drop which remains stuck to the impacting point, the glass bead bounces off leaving the armored puddle.

The second consequence of this bridging effect is that opening a bare hole of width $e^{*}$ in a double armor requires a surface extension twice as large as the one needed for a single armor and therefore corresponds to a threshold kinetic energy twice larger. As a result, the threshold velocity of coalescence for a double armor is larger than the one for a single armor by a factor of $\sqrt{2}$.

$V_{2}$, the measured threshold velocity of coalescence for the double armor situation, is reported as a function of the expression of Eq. (3) in Fig. 8 (open symbols). Here also, we observe a very good agreement. Indeed, besides the fact that the experimental data all collapses around the same line, the slope ratio between $V_{1}$ and $V_{2}$ equals $11.1 / 7.9 \approx 1.40$ very close to the predicted value of $\sqrt{2}$. Our previous understanding of the single armor coalescence is therefore confirmed by the double armor case.

\section{CONCLUSION}

The coalescence of particle armored interfaces can be forced under impact. This phenomenon is associated to the opening of a hole of a critical size, as previously reported for impacts of liquid marbles onto solid surfaces. ${ }^{10,37}$ Yet, contrary to the impact of liquid marbles onto solid substrates where the particles mobility remains unknown, here the shape and critical size of the hole required for the coalescence can be estimated. This leads to the elaboration of a coalescence criterion that gives the threshold velocity of coalescence of single armor (a bare drop impacting an armored puddle) which is in good agreement with the experiments. This quantitative criterion also applies to determine the threshold velocity of a double armor (an armored drop impacting an armored puddle), considering that the particles are mutualized between the two interfaces. The threshold velocity of a double armor is found to be larger than the one of a single one by a factor of $\sqrt{2}$ in quantitative agreement with the experiments.

\section{ACKNOWLEDGMENTS}

This work has been supported by CNES (convention CNES/CNRS No. 103954). We thank Michèle Adler, Florence Rouyer, Guillaume Lagubeau, and David Quéré for fruitful discussions. We thank Nicholas Blanchard for his careful reading of the manuscript.

${ }^{1}$ W. Ramsden, "Separation of solids in the surface-layers of solutions and suspensions," Proc. R. Soc. London 72, 156 (1903).

${ }^{2}$ S. U. Pickering, “Emulsions,” J. Chem. Soc. 91, 2001 (1907).

${ }^{3}$ B. D. Johnson and R. C. Cooke, "Generation of stabilized microbubbles in seawater," Science 213, 209 (1981).

${ }^{4}$ S. I. Kam and W. R. Rossen, "Anomalous capillary pressure, stress, and stability of solids-coated bubbles," J. Colloid Interface Sci. 213, 329 (1999).

${ }^{5}$ M. Abkarian, A. B. Subramaniam, S. H. Kim, R. J. Larsen, S. M. Yang, and H. A. Stone, "Dissolution arrest and stability of particle-covered bubbles," Phys. Rev. Lett. 99, 188301 (2007).

${ }^{6}$ A. B. Subramaniam, M. Abkarian, and H. A. Stone, "Controlled assembly of jammed colloidal shells on fluid droplets," Nature Mater. 4, 553 (2005).

${ }^{7}$ A. B. Subramaniam, M. Abkarian, L. Mahadevan, and H. A. Stone, "Non-spherical bubbles," Nature (London) 438, 930 (2005). 
${ }^{8}$ A. B. Subramaniam, M. Abkarian, L. Mahadevan, and H. A. Stone, "Mechanics of interfacial composite materials," Langmuir 22, 10204 (2006).

${ }^{9}$ P. Aussillous and D. Quéré, "Liquid marbles," Nature (London) 411, 924 (2001).

${ }^{10}$ P. Aussillous and D. Quéré, "Properties of liquid marbles," Proc. R. Soc. London, Ser. A 462, 973 (2006).

${ }^{11}$ G. McHale and M. I. Newton, "Liquid marbles: Principles and applications," Soft Matter 7, 5473 (2011).

${ }^{12}$ S. Fujii, P. D. Iddon, A. J. Ryan, and S. P. Armes, "Aqueous particulate foams stabilized solely with polymer latex particles," Langmuir 22, 7512 (2006).

${ }^{13}$ B. P. Binks and R. Murakami, "Phase inversion of particle-stabilized materials from foams to dry water," Nature Mater. 5, 865 (2006).

${ }^{14}$ A. Stocco, E. Rio, B. P. Binks, and D. Langevin, “Aqueous foams stabilized solely by particles," Soft Matter 7, 1260 (2011).

${ }^{15}$ A. Stocco, F. Garcia-Moreno, I. Manke, J. Banhart, and D. Langevin, "Particle-stabilised foams: Structure and aging," Soft Matter 7, 631 (2011).

${ }^{16}$ A. Cervantes Martinez, E. Rio, G. Delon, A. Saint-Jalmes, D. Langevin, and B. P. Binks, "On the origin of the remarkable stability of aqueous foams stabilised by nanoparticles: Link with microscopic surface properties," Soft Matter 4, 1531 (2008).

${ }^{17}$ T. S. Horozov, "Foams and foam films stabilised by solid particles," Curr. Opin. Colloid Interface Sci. 13, 134 (2008).

${ }^{18}$ L. Forny, I. Pezron, K. Saleh, P. Guigon, and L. Komunjer, "Storing water in powder form by self-assembling hydrophobic silica nanoparticles," Powder Technol. 171, 15 (2007).

${ }^{19}$ H. Brunner, D. Schutte, and F.-T. Schmitz, "Predominantly aqueous compositions in a fluffy powdery form approximating powdered solids behaviour and process for doing the same," patent DE1467023, 1969.

${ }^{20}$ S. Hasenzahl, A. Gray, E. Walzer, and A. Braunagel, "Dry water for the skin," SOEFW J. 131, 1 (2005).

${ }^{21}$ E. Bormashenko and A. Musin, "Revealing of water surface pollution with liquid marbles," Appl. Surf. Sci. 255, 6429 (2009).

22 J. Tian, T. Arbatan, X. Li, and W. Shen, "Liquid marble for gas sensing," Chem. Commun. 46, 4734 (2010).

${ }^{23}$ G. McHale, D. L. Herbertson, S. J. Elliott, N. J. Shirtcliffe, and M. I. Newton, "Electrowetting of nonwetting liquids and liquid marbles," Langmuir 23, 918 (2007).

${ }^{24}$ L. C. Gao and T. McCarthy, "Ionic liquid marbles," Langmuir 23, 10445 (2007).

${ }^{25}$ N. D. Denkov, B. I. Ivanov, P. A. Kralchevsky, and D. T. Wasan, "A possible mechanism of stabilization of emulsions by solid particles," J. Colloid Interface Sci. 150, 589 (1992).

${ }^{26}$ G. Kaptay, "Interfacial criteria for stabilization of liquid foams by solid particles," Colloids Surf., A 230, 67 (2003).

${ }^{27}$ T. S. Horozov, R. Aveyard, and J. H. Clint, "Particle zips: Vertical emulsion films with particle monolayers at their surfaces," Langmuir 21(6), 2330 (2005).

${ }^{28}$ G. Morris, M. R. Pursell, S. J. Neethling, and J. J. Cilliers, "The effect of particle hydrophobicity, separation distance and packing patterns on the stability of a thin film," J. Colloid Interface Sci. 327, 138 (2008).

${ }^{29}$ J. Eggers, J. R. Lister, and H. A. Stone, "Coalescence of liquid drops,” J. Fluid Mech. 401, 293 (1999).

${ }^{30}$ E. J. Stancik, M. Kouhkan, and G. G. Fuller, "Coalescence of particle-laden fluid interfaces," Langmuir 20, 90 (2004).

${ }^{31}$ S. Ata, "Coalescence of bubbles covered by particles," Langmuir 24, 6085 (2008).

${ }^{32}$ S. Ata, "The detachment of particles from coalescing bubble pairs," J. Colloid Interface Sci. 338, 558 (2009).

${ }^{33}$ S. A. Kulkarni, S. B. Ogale, and K. P. Vijayamohanan, "Tuning the hydrophobic properties of silica particles by surface silanization using mixed self-assembled monolayers," J. Colloid Interface Sci. 318, 372 (2008).

${ }^{34}$ E. Bormashenko, R. Pogreb, G. Whyman, and A. Musin, "Surface tension of liquid marbles," Colloids Surf., A 351, 78 (2009).

${ }^{35}$ C. Planchette, E. Lorenceau, and A. L. Biance, "Surface wave on a particle raft," Soft Matter 8, 2444 (2012).

${ }^{36}$ Y. Renardy, S. Popinet, L. Duchemin, M. Renardy, S. Zaleski, C. Josserand, M. A. Drumright-Clarke, D. Richard, C. Clanet, and D. Quéré, "Pyramidal and toroidal water drops after impact on a solid surface," J. Fluid Mech. 484, 69 (2003).

${ }^{37}$ C. Planchette, A. L. Biance, and E. Lorenceau, "Transition of liquid marble impacts onto solid surfaces," Europhys. Lett. 97, 14003 (2012).

${ }^{38}$ H. M. Princen, "Shape of a fluid drop at a liquid-liquid interface," J. Colloid Sci. 18, 178 (1963).

${ }^{39}$ F. Bashforth and J. C. Adams, An Attempt to Test the Theories of Capillary Action (University Press, Cambridge, 1883).

${ }^{40}$ C. Clanet, C. Beguin, D. Richard, and D. Quere, "Maximal deformation of an impacting drop," J. Fluid Mech. 517, 199 (2004).

${ }^{41}$ D. Vella, P. Aussillous, and L. Mahadevan, "Elasticity of an interfacial particle raft," Europhys. Lett. 68, 212 (2004).

${ }^{42}$ A. L. Biance, F. Chevy, C. Clanet, G. Lagubeau, and D. Quere, "On the elasticity of an inertial liquid shock," J. Fluid Mech. 554, 47 (2006).

${ }^{43}$ M. M. Bandi, T. Tallinen, and L. Mahadevan, "Shock-driven jamming and periodic fracture of particulate rafts," EPL 96, 36008 (2011).

${ }^{44}$ D. Vella, H. Y. Kim, P. Aussillous, and L. Mahadevan, "Dynamics of surfactant-driven fracture of particle rafts," Phys. Rev. Lett. 96, 178301 (2006).

${ }^{45}$ N. P. Ashby, B. P. Binks, and V. N. Paunov, "Bridging interaction between a water drop stabilised by solid particles and a planar oil/water interface," Chem. Commun. 2004, 436.

${ }^{46}$ E. J. Stancik and G. G. Fuller, "Connect the drops: Using solids as adhesives for liquids," Langmuir 20, 4805 (2004).

${ }^{47}$ T. S. Horozov and B. P. Binks, "Particle-stabilized emulsions: A bilayer or a bridging monolayer?," Angew. Chem., Int. Ed. Engl. 45, 773 (2006).

${ }^{48}$ H. Xu, M. Lask, J. Kirkwood, and G. Fuller, "Particle bridging between oil and water interfaces," Langmuir 23, 4837 (2007). 